TRANSACTIONS OF THE

AMERICAN MATHEMATICAL SOCIETY

Volume 177, March 1973

\title{
A SECOND QUADRANT HOMOTOPY SPECTRAL SEQUENCE
}

BY

\author{
A. K. BOUSF IELD AND D. M. KAN( $\left.{ }^{1}\right)$
}

\begin{abstract}
For each cosimplicial simplicial set with basepoint, the authors construct a homotopy s Dectral sequence generalizing the usual spectral sequence for a second quadrant double chain complex. For such homotopy spectral sequences, a uniqueness theore $m$ and a general multiplicative pairing are established. This machinery is used elsewhere to show the equivalence of various unstable Adams spectral sequences and to construct for them certain composition pairings and Whitehead products.
\end{abstract}

1. Introduction. The purpose of this paper is

(i) to show that there is sucb a thing as "the" (bomotopy) spectral sequence of a cosimplicial simplicial set with base point (resp. cosimplicial simplicial group), and

(ii) to construct for these spectral sequences a natural pairing.

Our prime example is "the unstable Adams spectral sequence" obtained in [1] by a different method. The above results will be applied in [2] to establish for that spectral sequence smash and composition pairings and Whitehead products.

We start with observing (in $\$ 2$ ) that the category of cosimplicial simplicial abelian groups is equivalent with the category of second quadrant double chain complexes and then devote the next six sections $(\S 3-\S 8)$ to formulating and proving

I. Let $\mathbf{X}$ be a cosimplicial simplicial set with base point and let $\pi_{t}^{\prime} \mathbf{X}^{\boldsymbol{s}}$ denote its normalized bomotopy groups, i.e.

$$
\pi_{t}^{\prime} \mathrm{X}^{s}=\pi_{t} \mathrm{X}^{s} \cap \operatorname{ker} s^{0} \cap \ldots \cap \operatorname{ker} s^{s-1} .
$$

Then there is (in a sense which will be made precise in $\$ 7$ ) a unique natural spectral sequence $\left\{E_{r} \mathbf{X}\right\}$ with

$$
\begin{aligned}
E_{1}^{s, t} \mathbf{X} & =\pi_{t}^{\prime} \mathbf{X}^{s} & & \text { for } t>s \geq 0 \\
& =0 & & \text { otherwise, }
\end{aligned}
$$

Received by the editors February 29, 1972.

AMS (MOS) subject classifications (1970). Primary 18G30, 18G40, 55 J10, 55H05.

Key words and phrases. Homotopy spectral sequence, simplicial set, cosimplicial object.

(1) This research was partially supported by NSF GP 8885 and NSF GP 23122. 
which, if $\mathbf{X}$ is a cosimplicial simplicial abelian group, coincides for $t>s \geq 0$ with the spectral sequence of the associated second quadrant double chain complex.

I'. Let $\mathbf{B}$ be a cosimplicial simplicial group. Then there is (in a sense which will be made precise in $\$ 4)$ a unique natural spectral sequence $\left\{E_{r} \mathbf{B}\right\}$ with

$$
\begin{aligned}
E_{1}^{s, t} \mathbf{B} & =\pi_{t}^{\prime} \mathbf{B}^{s} & & \text { for } t \geq s \geq 0, \\
& =0 & & \text { otherwise, }
\end{aligned}
$$

which, if $\mathbf{B}$ is abelian, coincides for $t \geq s \geq 0$ with the spectral sequence of the associated double chain complex.

The remainder of the paper is devoted to proving

II. Let $\mathbf{X}$ and $\mathbf{Y}$ be cosimplicial simplicial sets with base point. Then the pairing

$$
\begin{aligned}
\pi_{t}^{\prime} \mathrm{X}^{s} \wedge \pi_{t}^{\prime}, \mathrm{Y}^{s^{\prime}} & \stackrel{f}{\rightarrow} \pi_{t}^{\prime} \mathbf{X}^{s+s^{\prime}} \wedge \pi_{t^{\prime}}^{\prime} \mathrm{Y}^{s+s^{\prime}} \\
& \rightarrow \pi_{t+t^{\prime}}^{\prime}\left(\mathrm{X}^{s+s^{\prime}} \wedge \mathrm{Y}^{s+s^{\prime}}\right)=\pi_{t+t^{\prime}}^{\prime}(\mathrm{X} \wedge \mathrm{Y})^{s+s^{\prime}}
\end{aligned}
$$

(where $f$ denotes a graded version of the cosimplicial Alexander-Whitney map) induces a pairing of spectral sequences

$$
E_{r}^{s, t} \mathrm{X} \wedge E_{r}^{s^{\prime}, t^{\prime}} \mathrm{Y} \rightarrow E_{r}^{s+s^{\prime}, t+t^{\prime}}(\mathrm{X} \wedge \mathrm{Y})
$$

II'. A similar result for the group case.

The definition of the spectral sequences, the proof of their uniqueness and the demonstration that the above pairing of $E_{1}$-terms induces a pairing of spectral sequences, all involve certain universal examples which are introduced in $\$ 5$.

Our notation and conventions for cosimplicial objects will be as in [1].

2. Preliminaries.

2.1. The normalization of a simplicial abelian group. We recall from [4], [5] that for a simplicial abelian group $A$ the normalization $N_{*} A$ is the chain complex, trivial in dimension $<0$, defined in the following two equivalent manners:

$$
N_{n} A=A_{n} \cap \operatorname{ker} d_{1} \cap \cdots \cap \operatorname{ker} d_{n}
$$

with boundary map induced by the "remaining" face operator $d_{0}$, or

$$
N_{n} A=A_{n} /\left(\mathrm{im} s_{0} \oplus \cdots \oplus \mathrm{im} s_{n-1}\right)
$$

with boundary map given by $\partial=\sum_{i=0}^{n}(-1)^{i} d_{i}: N_{n} A \rightarrow N_{n-1} A$.

Both definitions yield the same identification $H_{q} N_{*} A=\pi_{q} A, q \geq 0$. Moreover, the functor $N_{*}$ is an equivalence between the categories of simplicial abelian groups and of abelian chain complexes which are trivial in dimension $<0$.

Dualizing this we get 
2.2. The normalization of a cosimplicial abelian group. For a cosimplicial abelian group $\mathbf{A}$ we define its normalization $N^{*} \mathbf{A}$ as the cochain complex, trivial in dimension $<0$, with

$$
N^{n} \mathbf{A}=\mathbf{A}^{n} \cap \operatorname{ker} s^{0} \cap \cdots \cap \operatorname{ker} s^{n-1}
$$

and coboundary map given by $\delta=\sum_{i=0}^{n}(-1)^{i} d^{i}: N^{n-1} \mathbf{A} \rightarrow N^{n} \mathbf{A}$. Clearly, the functor $N^{*}$ is an equivalence between the categories of cosimplicial abelian groups and of abelian cochain complexes which are trivial in dimension $<0$.

2.3. A nonabelian remark. Definition 2.2 also makes sense when applied to a cosimplicial (but not necessarily abelian) group; in that case, however, the coboundary maps need not be homomorphisms.

Finally we consider

2.4. The double normalization of a cosimplicial simplicial abelian group. If $\mathbf{A}$ is a cosimplicial simplicial abelian group, then $N^{*} N_{*} \mathbf{A}=N_{*} N^{*} \mathbf{A}$, and hence it makes sense to talk about its "double" normalization. Clearly, this double normalization functor is an equivalence between the categories of cosimplicial simplicial abelian groups and of second quadrant double chain complexes.

3. The spectral sequence of a cosimplicial simplicial abelian group. The spectral sequence $\left\{E_{r} \mathbf{A}\right\}$ of a cosimplicial simplicial abelian group $\mathbf{A}$ can be defined as the spectral sequence of its double normalization (2.4) $N^{*} N_{*} \mathbf{A}=$ $N^{*} N_{*} \mathbf{A}$, i.e., one forms the total chain complex $\left(T \mathbf{A}, d_{T}\right)$ given by

$$
\begin{aligned}
& T_{q} \mathbf{A}=\bigoplus_{t-s=q} N^{s} N_{t} \mathbf{A}, \\
& d_{T} b=\delta b+(-1)^{s} \partial b \quad \text { for } b \in N^{s} N_{t} \mathbf{A},
\end{aligned}
$$

filters this by putting $F^{s} T \mathbf{A}=\bigoplus_{i \geq s} N^{i} N_{*} \mathbf{A}$, and then takes the associated spectral sequence.

For our purposes, however, we need

3.1. A more explicit definition. Denote by $\pi_{t}^{\prime} \mathrm{A}^{s} \subset \pi_{t} \mathrm{~A}^{s}$ the normalized bomotopy group, i.e. the subgroup $\pi_{t} N^{s} \mathbf{A}=H_{t} N_{*} N^{s} \mathbf{A}=N^{s} H_{t} N_{*} \mathbf{A}=N^{s} \pi_{t} \mathbf{A} \subset$ $\pi_{t} A^{s}$, and define "relations", i.e. not always defined multivalued functions $d_{r}: \pi_{t}^{\prime} \mathbf{A}^{s}-\pi_{t+r-1}^{\prime} \mathbf{A}^{s+r}, r \geq 1$, by putting $d_{r} b=c$ whenever one can get from $b$ to $c$ by diagram chasing, i.e. whenever there are elements $b_{i} \in N^{s+i} N_{t+i} A$ $(0 \leq i<r)$ such that $b_{0} \in b, \delta b_{r-1} \in c$ and $\delta b_{i-1}=(-1)^{s+i+1} \partial b_{i}(0<i<r)$. One readily verifies that these relations have the following properties:

(i) naturality: if $b \in \pi_{t}^{\prime} \mathrm{A}^{s}$ and $c \in \pi_{t+r-1}^{\prime} \mathrm{A}^{s+r}$ are such that $\cdot d_{r} b=c$ and if $f: \mathbf{A} \rightarrow \mathbf{B}$ is a cosimplicial map, then $d_{r} f_{*} b=f_{*} c$,

(ii) additivity: if $b, b^{\prime} \in \pi_{t}^{\prime} \mathrm{A}^{s}$ and $c, c^{\prime} \in \pi_{t+r-1}^{\prime} \mathrm{A}^{s+r}$ are such that $d_{r} b=$ $c$ and $d_{r} b^{\prime}=c^{\prime}$, then $d_{r}\left(b-b^{\prime}\right)=c-c^{\prime}$, 
(iii) $d_{1}$ is the function $\sum_{i=0}^{s+1}(-1)^{i} d_{*}^{i}: \pi_{t}^{\prime} \mathbf{A}^{s} \rightarrow \pi_{t}{ }^{\prime} \mathrm{A}^{s+1}$,

(iv) the domain of definition of $d_{r}(r>1)$ is the kernel of $d_{r-1}$ : if $b \in \pi_{t}^{\prime} \mathrm{A}^{s}$, then $d_{r} b=c$ for some $c$ if and only if $d_{r-1} b=0$,

(v) the indeterminacy of $d_{r}(r>1)$ is the image of $d_{r-1}$ : if $b \in \pi_{t}^{\prime} \mathbf{A}^{s}$, then $d_{r} 0=b$ if and only if $d_{r-1} a=b$ for some $a$,

(vi) the $d_{r}$ are "differentials": if $b \in \pi_{t}^{\prime} \mathrm{A}^{s}$ and $c \in \pi_{t+r-1}^{\prime} \mathrm{A}^{s+r}$ are such that $d_{r} b=c$, then $d_{r} c=0$.

In view of these properties one now can obtain the spectral sequence $\left\{E_{r} \mathbf{A}\right\}$ by putting

$$
\begin{aligned}
& E_{1}^{s, t} \mathbf{A}=\pi_{t}^{\prime} \mathbf{A}^{s} \\
& E_{r}^{s, t} \mathbf{A}=\left(\pi_{t}^{\prime} \mathbf{A}^{s} \cap \operatorname{ker} d_{r-1}\right) /\left(\pi_{t}^{\prime} \mathbf{A}^{s} \cap \mathrm{im} d_{r-1}\right), \quad r>1,
\end{aligned}
$$

where

$$
\operatorname{ker} d_{r-1}=\left\{b \mid d_{r-1} b=0\right\}, \quad \text { im } d_{r-1}=\left\{c \mid d_{r-1} b=c \text { for some } b\right\}
$$

and defining the differentials $d_{r}: E_{r}^{s, t} \mathbf{A} \rightarrow E_{r}^{s+r, t+r-1} \mathbf{A}$ as the homomorphisms induced by the relations $d_{r}$. Moreover one clearly has $E_{r+1}^{s, t} \mathrm{~A} \subset E_{r}^{s, t} \mathrm{~A}$, for $r>s$, and hence the $E_{\infty}$-term is given by $E_{\infty}^{s, t} \mathrm{~A}=\bigcap_{r>s} E_{r}^{s, t} \mathrm{~A}$.

We end with observing that all this can be slightly generalized in

3.2. The augmented case. If $\mathbf{A}$ is augmented [1, \$9], then one can augment the above spectral sequence, i.e. define a natural filtration

$$
\cdots \subset F_{t}^{q+1} \mathbf{A} \subset F_{t}^{q} \mathbf{A} \subset \cdots \subset F_{t}^{0} \mathbf{A}=\pi_{t} \mathbf{A}^{-1}
$$

together with homomorphisms

$$
F_{t}^{q} \mathbf{A} \stackrel{e}{\rightarrow} E_{\infty}^{q, t+q} \mathbf{A}, \quad q \geq 0
$$

such that ker $e_{q}=F_{t}^{q+1} \mathrm{~A}$, as follows: Let $e_{q}: \pi_{t} \mathrm{~A}^{-1} \rightarrow \pi_{t+q}^{\prime} \mathrm{A}^{q}$ denote the relation obtained by putting $e_{q} a=b$ whenever there are elements $a_{-1} \in N_{t} \mathrm{~A}^{-1}$ and $a_{i} \epsilon$ $N_{t+i+1} N^{i} \mathrm{~A}(0 \leq i<q)$ such that $a_{-1} \in a, \delta a_{q-1} \in b$ and $\delta a_{i-1}=(-1)^{i+1} \partial a_{i}$ $(0<i<q)$. As in 3.1, these relations have the properties:

(i) naturality,

(ii) additivity,

(iii) $e_{0}$ is the function $d_{*}^{0}: \pi_{t} \mathbf{A}^{-1} \rightarrow \pi_{t} \mathbf{A}^{0}=\pi_{t}{ }^{\prime} \mathbf{A}^{0}$,

(iv) the domain of definition of $e_{q}(q>0)$ is the kermel of $e_{q-1}$,

(v) the indeterminacy of $e_{q}(q>0)$ is the image of $d_{q}$,

(vi) the image of $e_{q}$ contains only "infinite cycles", i.e. if $a \in \pi_{t} \mathbf{A}^{-1}$ and

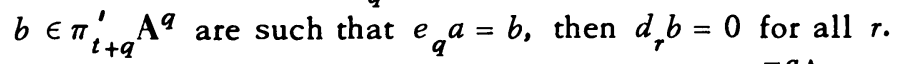

In view of these properties we can now put $F_{t}^{q} \mathbf{A}=\pi_{t} \mathbf{A}^{-1} \cap$ ker $e_{q-1}, q \geq 0$, 
and observe that each relation $e_{q}$ then induces a homomorphism $F_{t}^{q} \mathbf{A} \stackrel{e_{q}}{\rightarrow} E_{\infty}^{q, t+q} \mathbf{A}$, $q \geq 0$, whose kernel is exactly $F_{t}^{q+1} \mathbf{A}$.

4. The spectral sequence of a cosimplicial simplicial group. We now formulate our first result which, roughly speaking, states that for cosimplicial simplicial groups there is a unique (natural) spectral sequence generalizing the one of $\$ 3$, i.e. which for cosimplicial simplicial abelian groups coincides with (part of) the spectral sequence of $\S 3$. To be precise we have

4.1. Theorem. Let $\mathbf{B}$ be an augmented cosimplicial simplicial group and let $\pi_{t}^{\prime} \mathbf{B}^{s}=N^{s} \pi_{t} \mathbf{B} \subset \pi_{t} \mathbf{B}^{s}$. Then there are unique relations

$$
\begin{array}{ll}
d_{r}: \pi_{t}^{\prime} \mathrm{B}^{s} \rightarrow \pi_{t+r-1}^{\prime} \mathbf{B}^{s+r}, & r \geq 1, t \geq s \geq 0 \\
e_{q}: \pi_{t} \mathbf{B}^{-1} \rightarrow \pi_{t+q}^{\prime} \mathbf{B}^{q}, & q \geq 0, t \geq 0,
\end{array}
$$

which

(i) bave properties (i) through (vi) of 3.1 and 3.2 (except that we do not insist on the additivity of $d_{r}$ for $t=s=0, r \geq 1$; but we instead require $d_{r}$ : $\pi_{0}^{\prime} B^{0}-\pi_{r-1}^{\prime} \mathbf{B}^{r}$ to be "crossed-additive" for $r \geq 2$, i.e. if $b, b^{\prime} \in \pi_{0}^{\prime} \mathbf{B}^{0}$ and $c$, $c^{\prime} \in \pi_{r-1}^{\prime} \mathbf{B}^{r}$ are such that $d_{r} b=c$ and $d_{r} b^{\prime}=c^{\prime}$, then $d_{r}\left(b-b^{\prime}\right)=c-\left(b-b^{\prime}\right)^{\prime}$ where the left action of $\operatorname{ker} d_{1} \subset \pi_{0}^{\prime} \mathbf{B}^{0}$ on $\pi_{r-1}^{\prime} \mathbf{B}^{r}$. is induced by the natural homomorphism ker $d_{1} \rightarrow \pi_{0} \mathbf{B}^{r}$ and the left action of $\pi_{0} \mathbf{B}^{r}$ on $\pi_{r-1}^{\prime} \mathbf{B}^{r}$ ), and

(ii) for abelian $\mathbf{B}$, coincide with the relations $d_{r}$ and $e_{q}$ of $\S 3$.

This allows us to define

4.2. The spectral sequence of an (augmented) cosimplicial simplicial group. The spectral sequence $\left\{E_{r} B\right\}$ of a cosimplicial simplicial group $\mathbf{B}$ is the spectral sequence obtained by putting

$$
\begin{aligned}
E_{1}^{s, t} \mathbf{B} & =\pi_{t}^{\prime} \mathbf{B}^{s}, \quad t \geq s \geq 0, \\
& =0, \quad \text { otherwise, } \\
E_{r}^{s, t} \mathbf{B} & =\left(E_{1}^{s, t} \mathbf{B} \cap \operatorname{ker} d_{r-1}\right) /\left(E_{1}^{s, t} \mathbf{B} \cap \operatorname{im} d_{r-1}\right), \\
E_{\infty}^{s, t} \mathbf{B} & =\bigcap_{r>s} E_{r}^{s, t} \mathbf{B}
\end{aligned}
$$

with, as differentials, the homomorphisms $d_{r}: E_{r}^{s, t} \mathbf{B} \rightarrow E_{r}^{s+r, t+r-1} \mathbf{B}$ induced by the relations $d_{r}$ of 4.1 .

Furthermore if $\mathbf{B}$ is augmented, then, as in 3.2, we can augment this spectral sequence by putting $F_{t}^{q} \mathbf{B}=\pi_{t} \mathbf{B}^{-1} \cap \operatorname{ker} e_{q-1}, q \geq 0$, and observing that the relations $e_{q}$ of 4.1 then induce homomorphisms $F_{t}^{q} \mathbf{B} \stackrel{e_{q}}{\rightarrow} E_{\infty}^{q, t+q} \mathbf{B}$ whose kernel is exactly $F_{t}^{q+1} \mathbf{B}$. 
Clearly if $\mathbf{B}$ is abelian this (augmented) spectral sequence coincides for $t-s \geq 0$ with the one of $\$ 3$, justifying the use of the same notation.

Also, $[2, \S 5]$, readily implies

4.3. Corollary. The above (augmented) spectral sequence is naturally equivalent with the one of $[2, \S 11]$.

4.4. Remark. The above spectral sequence is "fringed" in dimension 0 in the sense of $[1, \S 4]$, i.e. $E_{r} \mathbf{B}=H\left(E_{r-1} \mathbf{B}, d_{r-1}\right)$ except possibly when $t-s=0$, when one may have $E_{r} \mathbf{B} \subset H\left(E_{r-1} \mathbf{B}, d_{r-1}\right)$ instead.

The proof of Theorem 4.1 will be given in $\$ 6$.

5. Universal examples for the $d_{r}$ and $e_{q}$. In this section we discuss certain cosimplicial objects $\mathbf{D}_{(r, s, t)}$ and $\mathbf{E}_{(q, t)}$ which will turn out to be universal examples for the $d_{r}$ and $e_{q}$. They are the main tool in the proof of Theorem 4.1 ( $\$ 6)$ and will also be used to prove the existence of pairings $(\S 10)$.

5.1. The universal example $\mathbf{D}_{(r, s, t)}$. For $r \geq 1$ and $s \geq 0, \mathbf{D}_{(r, s, s)}$ will be the cosimplicial simplicial set with base point where each $\mathbf{D}_{(r, s, s)}^{n}$ is obtained from the standard simplex $\Delta[n][5, \mathrm{p} .14]$ by

(i) collapsing the $(s-1)$-skeleton to a base point (for $s=0$ this should be interpreted as "taking the disjoint union with a base point"), and

(ii) taking the $(s+r-1)$-skeleton,

and where the cofaces and codegeneracies are induced by the standard maps 15, p. 14]

$$
\Delta[n-1] \stackrel{\delta_{i}}{\rightarrow} \Delta[n], \quad \Delta[n+1] \stackrel{\sigma_{i}}{\rightarrow} \Delta[n], \quad 0 \leq i \leq n .
$$

For $t \geq s \geq 0, \mathbf{D}_{(r, s, t)}$ then is obtained from $\mathbf{D}_{(r, s, s)}$ by application (in the sense of $[1, \S 9]$ of the $(t-s)$-fold suspension functor [5, p. 124].

Note that this definition also makes sense if $r=\infty$ and that

$$
\mathbf{D}_{(\infty, s, t)} / \mathbf{D}_{(r, s, t)}=\mathbf{D}_{(\infty, s+r, t+r)} .
$$

Let $Z$ be the "free abelian group on" functor [1], i.e. the functor which assigns to a simplicial set with base point $X$ the simplicial (free) abelian group generated by the simplices of $X$ with the base point (and its degeneracies) put equal to the identity. Then we have

5.2. Properties of $Z \mathbf{D}_{(\infty, s, t)}$. (i) $\pi_{t}^{\prime} Z \mathbf{D}_{(\infty, s, t)}^{s} \approx Z$ and is generated by the nondegenerate t-simplex of $\mathbf{D}_{(\infty, s, t)}^{s}$,

(ii) $\pi_{n}^{\prime} Z \mathbf{D}_{(\infty, s, t)}^{k}=0$ otherwise.

Proof. This follows readily from the fact that for any simplicial set with base point $X$ there is a natural isomorphism $\pi_{*} Z X \approx \widetilde{H}_{*} X$, where $\widetilde{H}_{*}$ denotes the reduced (integral) homology, and the observation that each $D_{(\infty, s, t)}^{k}$ has the homotopy type of a wedge of $t$-spheres. 
5.3. Properties of $Z \mathbf{D}_{(r, s, t)}$. (i) $\pi_{t}^{\prime} Z \mathbf{D}_{(r, s, t)}^{s} \approx Z$ and is generated by the nondegenerate $t$-simplex of $\mathbf{D}_{(r, s, t)}^{s}$ $\mathbf{D}_{(r, s, t)}^{s+r}$,

(ii) $\pi_{t+r-1}^{\prime} Z \mathbf{D}_{(r, s, t)}^{s+r} \approx Z$ and is generated by the collapsing map $\dot{\Delta}[t+r] \rightarrow$

(iii) $\pi_{n}^{\prime} Z \mathbf{D}_{(r, s, t)}^{k}=0$ otherwise, and

(iv) the differential $d_{r}$ in $E_{r} Z \mathbf{D}_{(r, s, t)}$ is an isomorphism

$$
E_{r}^{s, t}=\pi_{t}^{\prime} Z \mathbf{D}_{(r, s, t)}^{s} \stackrel{a_{r}}{\underset{\approx}{*}} E_{r}^{s+r, t+r-1}=\pi_{t+r-1}^{\prime} Z \mathbf{D}_{(r, s, t)}^{s+r}
$$

Proof. The first three parts follow readily trom 5.2 and (5.1a) while part (iv) is a simple calculation.

If $F$ denotes Milnor's "free group on" functor [3], i.e. the functor which assigns to a simplicial set with base point $X$ the simplicial (free) group generated by the simplices of $X$ with the base point (and its degeneracies) put equal to the identity, then we can for the $F \mathbf{D}_{(r, s, t)}$ formulate properties similar to 5.2 and 5.3 . To do this we need the notion of

5.4. The additive subgroup of $\pi_{n}^{\prime} F \mathbf{D}_{(r, s, t)}^{k}$. For $K \in S_{*}$ and $n \geq 1$ let $\pi_{n} F K C \pi_{n} F K$ denote the subgroup consisting of all $u \in \pi_{n} F K$ such that $f_{12^{*}} u=$ $f_{1^{*}} u+f_{2^{*}} u$ where $f_{1}, f_{2}, f_{12}: F K \rightarrow F(K \bigvee K)$ are the homomorphisms which respectively send $x \in K$ to $x_{1}, x_{2}, x_{1} x_{2} \in F(K \bigvee K)$ (with $x_{1}$ denoting $x$ in the $i$ th $K$ ). It is easy to show that

(i) the inclusion $K \rightarrow F K$ induces a bomomorpbism $\pi_{n} K \rightarrow \tilde{\pi}_{n} F K$, and

(ii) for any $K, L \in S_{*}, \tilde{\pi}_{n} F(K \vee L) \approx \tilde{\pi}_{n} F K \oplus \tilde{\pi}_{n} F L$. If we put $\tilde{\pi}_{n}^{\prime} F D_{(r, s, t)}^{k}=$ $\tilde{\pi}_{n} F \mathbf{D}_{(r, s, t)}^{k} \cap \pi_{n}^{\prime} F \mathbf{D}_{(r, s, t)}^{k}$ then we can formulate

5.5. Properties of $F \mathbf{D}_{(r, s, t)}$ for $t>0$. (i) $\pi_{t}^{\prime} F \mathbf{D}_{(r, s, t)}^{s}=\pi_{t}^{\prime} F \mathbf{D}_{(r, s, t)}^{s} \approx Z$ and is generated by the nondegenerate t-simplex of $\mathbf{D}_{(r, s, t)}^{s}$; $\mathbf{D}_{(r, s, t)}^{s+r}$.

(ii) $\tilde{\pi}_{t+r-1}^{r} F \mathbf{D}_{(r, s, t)}^{s+r} \approx Z$ and is generated by the collapsing map $\dot{\Delta}[t+r] \rightarrow$

Proof. This follows easily using the fact that $\mathbf{D}_{(r, s, t)}^{k}$ is equivalent to a wedge of $t$-spheres and $(t+r-1)$-spheres.

5.6. Properties of $F \mathbf{D}_{(r, 0,0)}$ for $r \geq 2$. As in 4.1 (i) we view $\pi_{r-1}^{\prime} F D_{(r, 0,0)}^{r}$ as a left $\pi_{0}^{\prime} F \mathbf{D}_{(r, 0,0)}^{0}$ module. A straightforward computation then shows that there is a unique function $b: \pi_{0}^{\prime} F \mathbf{D}_{(r, 0,0)}^{0} \rightarrow \pi_{r-1}^{\prime} F \mathbf{D}_{(r, 0,0)}^{r}$ such that

(i) $b$ is a crossed homomorphism, i.e. $b(x+y)=b x+x(b y)$,

(ii) $b \xi_{*}=\xi_{*} b$ where $\xi: F \mathbf{D}_{(r, 0,0)} \rightarrow F \mathbf{D}_{(r, 0,0)}$ is the homomorphism sending $x \in \mathbf{D}_{(r, 0,0)}$ into $x^{2} \in F \mathbf{D}_{(r, 0,0)}$,

(iii) $d_{r} g_{*}=g_{*} d_{r}$ where $g: F \mathbf{D}_{(r, 0,0)} \rightarrow Z \mathbf{D}_{(r, 0,0)}$ is the abelianization.

We end with considering 
5.7. The universal example $\mathbf{E}_{(q, t)}$. For $q \geq 0$ and $t=0, \mathbf{E}_{(q, 0)}$ will be the augmented cosimplicial simplicial set with base point where each $\mathbf{E}_{(q, 0)}^{n}$ is obtained from the standard simplex $\Delta[n+1]$ by

(i) collapsing to a base point the image of $\Delta[n]$ under the map $\delta_{0}: \Delta[n] \rightarrow$ $\Delta[n+1]$, and

(ii) taking the $q$-skeleton, and where the operators $d^{i}$ and $s^{i}$ are induced by the standard maps

$$
\Delta[n] \stackrel{\delta_{i+1}}{\longrightarrow} \Delta[n+1], \quad \Delta[n+2] \stackrel{\sigma_{i+1}}{\longrightarrow} \Delta[n+1], \quad 0 \leq i \leq n .
$$

For $t>0, \mathbf{E}_{(q, t)}$ is then obtained from $\mathbf{E}_{(q, 0)}$ by application of the $t$-fold suspension functor.

One readily proves the following analogues of 5.3 and 5.5 .

5.8. Properties of $Z \mathrm{E}_{(q, t)}$. (i) $\pi_{t} Z \mathrm{E}_{(q, t)}^{-1} \approx Z$ and is generated by the nondegenerate $t$-simplex of $\mathrm{E}_{(q, t)}^{-1}$, $\mathbf{E}_{(q, t)}^{q}$,

(ii) $\pi_{t+q}^{\prime} Z \mathbf{E}_{(q, t)}^{q} \approx Z$ and is generated by the collapsing map $\dot{\Delta}[t+q+1] \rightarrow$

(iii) $\pi_{n}^{\prime} Z E_{(q, t)}^{k}=0$ otherwise, and

(iv) the bomomorphism $e_{q}$ (3.2) is an isomorphism

$$
F_{t}^{q}=\pi_{t} Z \mathrm{E}_{(q, t)}^{-1} \stackrel{e}{\stackrel{q}{\approx}} E_{\infty}^{q, t+q}=\pi_{t+q}^{\prime} Z \mathbf{E}_{(q, t)}^{q}
$$

5.9. Properties of $F \mathrm{E}_{(q, t)}$. (i) $\pi_{t} F \mathrm{E}_{(q, t)}^{-1} \approx Z$ and is generated by the nondegenerate t-simplex of $\mathrm{E}_{(q, t)}^{-1}$, and $\mathbf{E}_{(q, t)}^{q}$

(ii) $\pi_{t+q}^{\prime} F \mathrm{E}_{(q, t)}^{q} \approx Z$ and is generated by the collapsing map $\dot{\Delta}[t+q+1] \rightarrow$

6. Proof of Theorem 4.1. We now prove Theorem 4.1 and show at the same time that the $\mathbf{D}_{(r, s, t)}$ and $\mathbf{E}_{(q, t)}$ of $\$ 5$ are universal examples for the relations $d_{r}$ and $e_{q}$ of this theorem. To do this we define the relations $d_{r}$ and $e_{q}$ of Theorem 4.1 in terms of the $\mathbf{D}_{(r, s, t)}$ and $\mathbf{E}_{(q, t)}$ and then show that these relations have indeed all the desired properties.

6.1. Definition of the $d_{r}$ in terms of the $\mathbf{D}_{(r, s, t)}$. Choose a generator $i \epsilon$ $\pi_{t}^{\prime} F \mathbf{D}_{(r, s, t)}^{s}$, denote by $j \in \pi_{r-1}^{\prime} F \mathbf{D}_{(r, 0,0)}^{r}$ the element $d_{*}^{0} i-d_{*}^{1} i$ if $r-1$ and the element $b i$ (see 5.6) if $r \geq 2$, and for $t>0$ denote by $j \in \pi_{t+r-1}^{\prime} F \mathbf{D}_{(r, s, t)}^{s+r}$ the (in view of 5.5) unique element such that $d_{r} g_{*} i=g_{*} j$ where $g: F \mathbf{D}_{(r, s, t)} \rightarrow$ $Z \mathbf{D}_{(r, s, t)}$ is the abelianization. For any cosimplicial simplicial group $\mathbf{B}$ and elements $b \in \pi_{t}^{\prime} \mathbf{B}^{s}$ and $c \in \pi_{t+r-1}^{\prime} \mathbf{B}^{s+r}$ we then put $d_{r} b=c$ if and only if there is a bomomorphism $f: F \mathbf{D}_{(r, s, t)} \rightarrow \mathbf{B}$ such that $f_{*} i=b$ and $f_{*} j=c$.

Similarly we have 
6.2. Definition of the $e_{q}$ in terms of the $\mathbf{E}_{(q, t)}$. Choose a generator $i \epsilon$ $\pi_{t} F \mathbf{E}_{(q, t)}^{-1}$ and denote by $j \in \pi_{t+q}^{\prime} F \mathbf{E}_{(q, t)}^{q}$ the (in view of 5.9) unique element such that $e_{q} g_{*} i=g_{*} j$. For any augmented cosimplicial simplicial group $\mathbf{B}$ and elements $a \in \pi_{t} \mathbf{B}^{-1}$ and $b \in \pi_{t+q}^{\prime} \mathbf{B}^{q}$ we then put $e_{q}^{a=b}$ if and only if there is $a$ bomomorphism $f: F \mathbf{E}_{(q, t)} \rightarrow B$ such that $f_{*} i=a$ and $f_{*} j=b$.

Clearly both these definitions do not depend on the choice of the generator $i$.

One readily verifies that the relations defined above have properties 4.1 (ii), 3.1 (i), (ii) (modified as in 4.1(i)), and (iii) and 3.2 (i), (ii) and (iii) and that, conversely, they are uniquely determined by these properties. Properties 3.1 (iv) and 3.2 (iv) are equivalent to the following lemma which can be proved by calculations similar to the ones used in proving that a simplicial group satisfies the extension condition [5].

6.3. Lemma. Let $\mathbf{B}$ be an augmented cosimplicial simplicial group. A bomomorphism $f: F \mathbf{D}_{(r-1, s, t)} \rightarrow \mathbf{B}$ (resp. $\left.f: F \mathbf{E}_{(q-1, t)} \rightarrow \mathbf{B}\right)$ then can be extended to a bomomorphism $F \mathbf{D}_{(r, s, t)} \rightarrow \mathbf{B}$ (resp. $F \mathbf{E}_{(q, t)} \rightarrow \mathbf{B}$ ) if and only if $f_{*} j=0$.

The proof of Properties 3.1 (v) and 3.2 (v) is not hard. And finally Property 3.1 (vi) follows by naturality from the fact that (ignoring the augmentation) $\mathbf{D}_{(r, s, t)}=\mathbf{E}_{(s+r, t-s-1)} / \mathbf{E}_{(s, t-s-1)}$ and Lemma 6.6 below, while Property $3.2(v i)$ follows directly from this same lemma.

6.6. Lemma. $\pi_{n}^{\prime} F \mathrm{E}_{(q, t)}^{k}=0$ for $n-k<t$.

Proof. Let $d$ be the smallest integer such that $n<(d+1)(q+t)$. As each $\mathbf{E}_{(q, t)}^{k}$ has the homotopy type of a wedge of $(q+t)$-spheres, there is, by the Hilton-Milnor theorem [3], a functor $T$ from free abelian groups to abelian groups such that $\pi_{n} F \mathbf{E}_{(q, t)}=T H_{q+t} \mathbf{E}_{(q, t)}$. Moreover this functor is of degree $\leq d$ and hence, by [4, p. 233], $\pi_{n}^{\prime} F \mathbf{E}_{(q, t)}^{k}=0$ for $k>d q$. The desired result now follows readily.

7. The spectral sequence of a cosimplicial simplicial set with base point. Our next result is that, for cosimplicial simplicial sets with base point, there also is a unique (natural) spectral sequence generalizing the one of $\$ 3$. To be precise, we have

7.1. Theorem. Let $\mathbf{X}$ be an augmented cosimplicial simplicial set with base point and let $\pi_{t}^{\prime} \mathbf{X}^{s}=N^{s} \pi_{t} \mathbf{X} \subset \pi_{t} \mathbf{X}^{s}$. Then there are unique relations

$$
\begin{array}{ll}
d_{\cdot r}: \pi_{t}^{\prime} \mathrm{X}^{s} \rightarrow \pi_{t+r-1}^{\prime} \mathrm{X}^{s+r}, & r \geq 1, t>s \geq 0, \\
e_{q}: \pi_{t} \mathrm{X}^{-1} \rightarrow \pi_{t+q}^{\prime} \mathrm{X}^{q}, & q \geq 0, t>0,
\end{array}
$$

which 
(i) bave properties (i) through (vi) of 3.1 and 3.2 (except that we do not insist on the additivity of $d_{r}$ for $t=1, s=0, r \geq 1$; but instead we require such $d_{r}$ for $r \geq 2$ to be "crossed-additive", as in 4.1 (i)), and

(ii) for $\mathbf{X}$ an augmented cosimplicial simplicial abelian group, coincide with the relations $d_{r}$ and $e_{q}$ of $\$ 3$.

Thus we can define

7.2. The spectral sequence of an (augmented) cosimplicial simplicial set with base point. If $X$ is a cosimplicial simplicial set with base point, then its spectral sequence $\left\{E_{r} X\right\}$ with

$$
\begin{aligned}
E_{1}^{s, t} \mathbf{X} & =\pi_{t}^{\prime} \mathbf{Y}^{s}, & & t>s \geq 0 \\
& =0, & & \text { otherwise }
\end{aligned}
$$

can be defined as in 4.2. And, as in 4.2, an augmentation of $\mathbf{X}$ induces an augmentation of the spectral sequence.

If $\mathbf{X}$ is an augmented cosimplicial simplicial group, then, in view of Theorem 4.1, the above spectral sequence coincides for $t-s>0$ with the one of $\S 4$, justifying the use of the same notation.

Again $[2, \$ 5]$ readily implies

7.3. Corollary. The above (augmented) spectral sequence is naturally equiv. alent with the one of $[2, \$ 4]$.

7.4. Remark. The above spectral sequence is "fringed" in dimension 1 with the one of $[2, \S 4]$, i.e. $E_{r} \mathbf{X}=H\left(E_{r-1} \mathbf{X}, d_{r-1}\right)$ except possibly when $t-s=$ 1 , when one may have $E_{r} \mathbf{X} \subset H\left(E_{r-1} \mathbf{X}, d_{r-1}\right)$ instead.

8. Equivalence of the results of $\$ 4$ and $\$ 7$. Using the loop group functor $G$ and the classifying functor $\bar{W}[5]$ we will show

8.1. Proposition. Theorems 4.1 and 7.1 imply each other.

8.2. Proposition. Let $\mathbf{B}$ be an augmented cosimplicial simplicial group and let $\partial: \pi_{t+1} \bar{W} \mathbf{B}^{s} \approx \pi_{t} \mathbf{B}^{s}$ denote the boundary isomorphism of [5]. Then the "other" boundary isomorphisms

$$
\partial_{0 t}=(-1)^{t} \partial: \pi_{t+1} \bar{W} \mathbf{B}^{s} \approx \pi_{t} \mathbf{B}^{s}
$$

induce an augmented spectral sequence isomorphism, i.e. isomorphisms $\partial_{0 t}$ : $E_{r}^{s, t+1} \bar{W} \mathbf{B} \approx E_{r}^{s, t} \mathbf{B}, \partial_{0 t}: F_{t+1}^{q} \bar{W} \mathbf{B} \approx F_{t}^{q} \mathbf{B}$ which commute with the $d_{r}$ and $e_{q} \cdot$

8.3. Proposition. Let $\mathbf{X}$ be an augmented cosimplicial simplicial set with base point and let $\partial: \pi_{t+1} \mathbf{X}^{s} \approx \pi_{t} G \mathbf{X}^{s}$ denote the boundary isomorphism of [5]. 
Then the "other" boundary isomorphisms

$$
\partial_{0 t}=(-1)^{t} \partial: \pi_{t+1} \mathbf{X}^{s} \approx \pi_{t} G \mathbf{X}^{s}
$$

induce an augmented spectral sequence isomorphism, i.e. isomorphisms

$$
\partial_{0 t}: E_{r}^{s, t+1} \mathbf{X} \approx E_{r}^{s, t} G \mathbf{X}, \quad \partial_{0 t}: F_{t+1}^{q} \mathbf{X} \approx F_{t}^{q} G \mathbf{X}
$$

which commute with the $d_{r}$ and $e_{q}$.

These propositions follow readily from the basic properties of the functors $G$ and $\bar{W}[5]$ and the following observations which are not hard to verify.

(i) If $\mathbf{A}$ is an augmented cosimplicial simplicial abelian group, then the "other" boundary isomorphisms $\partial_{0 t}: \pi_{t+1} \bar{W} A^{s} \approx \pi_{t} \mathbf{A}^{s}$ induce isomorphisms $\partial_{0 t}$ : $\pi_{t+1}^{\prime} \bar{W} \mathbf{A}^{s} \approx \pi_{t}^{\prime} \mathbf{A}^{s}$ which commute with the relations $d_{r}$ and $e_{q}$ (of $\S 3$ ).

(ii) If $\mathbf{B}$ is an augmented cosimplicial simplicial group and $d_{r}$ and $e_{q}$ are relations on the $\pi_{t}^{\prime} \mathrm{B}^{s}$ and $\pi_{t} \mathbf{B}^{-1}$ satisfying 3.1 and 3.2 (i) through (vi), then the "other" boundary isomorphisms $\partial_{0 t}: \pi_{t+1} \bar{W} \mathbf{B}^{s} \approx \pi_{t} \mathbf{B}^{s}$ induce relations on the $\pi_{t+1}^{\prime} \bar{W} \mathbf{B}^{s}$ and $\pi_{t+1} \bar{W} \mathbf{B}^{-1}$ which also satisfy 3.1 and 3.2 (i) through (vi).

(iii) If $\mathbf{X}$ is an augmented cosimplicial set with base point and $d_{r}$ and $e_{q}$ are relations on the $\pi_{t+1}^{\prime} \mathrm{X}^{s}$ and $\pi_{t+1} \mathrm{X}^{-1}$ satisfying 3.1 and 3.2 (i) through (vi), then the "other" boundary isomorphisms $\partial_{0 t}: \pi_{t+1} \mathbf{X}^{s} \approx \pi_{t} G \mathbf{X}^{s}$ induce relations on the $\pi_{t}^{\prime} G \mathbf{X}^{s}$ and $\pi_{t} G \mathbf{X}^{-1}$ which also satisfy 3.1 and 3.2 (i) through (vi).

8.4. Remark on the proof of Theore $m$ 7.1. Instead of reducing it to Theorem 4.1, one can try to prove Theorem 7.1 directly using the arguments of $\$ 6$ with $\mathbf{D}_{(r, s, t)}$ and $\mathbf{E}_{(q, t)}$ instead of $F \mathbf{D}_{(r, s, t)}$ and $F \mathbf{E}_{(q, t)}$. One then runs into a slight problem because $X^{s}$ need not satisfy the extension condition, nor need the analogue of 6.3 be true. However, both these problems disappear if one replaces $\mathbf{X}$ by $\bar{W} G \mathbf{X}$.

9. Pairings (the abelian case). We end this paper with recalling the usual pairing of spectral sequences for augmented cosimplicial simplicial abelian groups (or equivalently (2.4) second quadrant double chain complexes) and showing the existence of a similar pairing for augmented cosimplicial simplicial groups and for augmented cosimplicial simplicial sets with base point. This section will deal with

9.1. The abelian group case. For any two simplicial abelian groups $A$ and $B$ we denote by $\pi_{t} A \wedge \pi_{t} B \stackrel{\wedge}{\rightarrow} \pi_{t+t}(A \otimes B)$ the pairing induced by the Eilenberg-Zilber map [4, p. 217] $N_{*} A \otimes N_{*} B \stackrel{g}{\rightarrow} N_{*}(A \otimes B)$, and recall that it is

(i) bilinear,

(ii) associative,

(iii) commutative (with sign $\left.(-1)^{t t^{\prime}}\right)$, i.e. $t_{*}(u \wedge v)=(-1)^{t t^{\prime}}(v \wedge u)$ for 
$u \in \pi_{t} A, v \in \pi_{t} \cdot B$ and $t: A \otimes B \rightarrow B \otimes A$ the twisting map.

For two augmented cosimplicial simplicial abelian groups $\mathbf{A}$ and $\mathbf{B}$ one then can define a pairing

$$
\pi_{t}^{\prime} \mathbf{A}^{s} \wedge \pi_{t}^{\prime}, \mathbf{B}^{s^{\prime}} \stackrel{\wedge}{\longrightarrow} \pi_{t+t}^{\prime}(\mathbf{A} \otimes \mathbf{B})^{s+s^{\prime}}
$$

as the map induced by the composition

$$
\pi_{t} \mathbf{A}^{s} \wedge \pi_{t}, \mathbf{B}^{s^{\prime}} \stackrel{f}{\rightarrow} \pi_{t} \mathbf{A}^{s+s^{\prime}} \stackrel{\wedge}{\rightarrow} \pi_{t} \mathbf{B}^{s+s^{\prime}} \stackrel{\wedge}{\rightarrow} \pi_{t+t^{\prime}}(\mathbf{A} \otimes \mathbf{B})^{s+s^{\prime}}
$$

where $f$ is the (graded) Alexander-Whitney map [4, p. 217] given by

$$
f(u, v)=\left((-1)^{t s^{\prime}} d^{s+s^{\prime}} \ldots d^{s+1} u, d^{s-1} \cdots d^{0} v\right) .
$$

A straightforward calculation then yields the following "well-known" result.

9.3. Theorem. The pairing (9.2) induces a pairing of spectral sequences

$$
E_{r}^{s, t} \mathbf{A} \wedge E_{r}^{s^{\prime}, t^{\prime}} \mathbf{B} \stackrel{\rightarrow}{\rightarrow} E_{r}^{s+s^{\prime}, t+t^{\prime}}(\mathbf{A} \otimes \mathbf{B}), \quad 1 \leq r \leq \infty,
$$

which is augmented, linear, associative and commutative (with sign $\left.(-1)^{(t-s)\left(t^{\prime}-s^{\prime}\right)}\right)$, i.e.

(i) the pairing on $E_{1}$ is the pairing (9.2),

(ii) for $u \in E_{r}^{s, t} \mathrm{~A}$ and $v \in E_{r}^{s^{\prime}, t}{ }^{\prime} \mathrm{B}(1 \leq r \leq \infty)$,

$$
d_{r}(u \wedge v)=\left(d_{r} u \wedge v\right)+(-1)^{t-s}\left(u \wedge d_{r} v\right)
$$

(iii) the pairing on $E_{r+1}$ is induced by the one on $E_{r}(1 \leq r<\infty)$ and the pairing on $E_{\infty}$ is induced by the ones on the $E_{r}(1 \leq r<\infty)$,

(iv) the pairing on $E_{\infty}$ is compatible with the augmentations, i.e., if $u \epsilon$ $F_{t}^{q} \mathrm{~A}$ and $v \in F_{t}^{q^{\prime}}, \mathbf{B}$, then $u \wedge v \in F_{t+t^{\prime}}^{q+q^{\prime}}(\mathbf{A} \otimes \mathbf{B})$ and $e_{q+q^{\prime}}(u \wedge v)=e_{q} \wedge e_{q}, v$,

(v) the pairing is bilinear,

(vi) the pairing is associative,

(vii) the pairing is commutative (with sign $\left.(-1)^{(t-s)\left(t^{\prime}-s^{\prime}\right)}\right)$ for $r \geq 2$.

9.4. A slight refinement. Let $\mathbf{A} \otimes^{\prime} \mathbf{B} \subset \mathbf{A} \otimes \mathbf{B}$ consist of the augmentation and all simplices of the form $\left(d^{s+s^{\prime}} \ldots d^{s+1} u \otimes d^{s-1} \cdots d^{0} v\right)$ where $u \in \mathbf{A}^{s}$, $v \in \mathbf{B}^{s^{\prime}}$ and $s, s^{\prime} \geq 0$. Then the pairing (9.2) can clearly be factored through a pairing

$$
\pi_{t}^{\prime} \mathbf{A}^{s} \wedge \pi_{t^{\prime}} \mathbf{B}^{s^{\prime}} \stackrel{\wedge^{\prime}}{\rightarrow} \pi_{t+t^{\prime}}^{\prime}\left(\mathbf{A} \otimes \otimes^{\prime} \mathbf{B}\right)^{s+s^{\prime}}
$$

and hence one has

9.5. Theorem. The pairing of 9.4 induces a pairing of spectral sequences

$$
E_{r}^{s, t} \mathbf{A} \wedge E_{r}^{s^{\prime}, t^{\prime}} \mathbf{B} \stackrel{\Lambda^{\prime}}{\rightarrow} E_{r}^{s+s^{\prime}, t+t^{\prime}}\left(\mathbf{A} \otimes{ }^{\prime} \mathbf{B}\right), \quad 1 \leq r \leq \infty,
$$


which is augmented, bilinear and associative (commutativity does not make sense).

10. Pairings (the other cases). First we consider

10.1. The sets with base point case. For any two simplicial sets with base point $X$ and $Y$ we denote by $\pi_{t} X \wedge \pi_{t}, Y \wedge \pi_{t+t},(X \wedge Y)$ the unique natural pairing such that, whenever $X$ and $Y$ are simplicial abelian groups, the following diagram commutes:

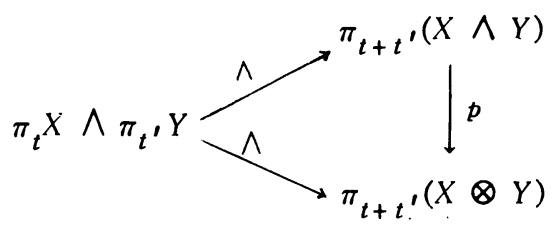

where $p(x, y)=x \otimes y$ for all $(x, y) \in X \wedge Y$. Clearly this pairing is

(i) linear in the first (second) variable whenever $t>0\left(t^{\prime}>0\right)$,

(ii) associative,

(iii) commutative (with sign $(-1)^{t t^{\prime}}$ ).

For two augmented cosimplicial simplicial sets with base point $\mathbf{X}$ and $\mathbf{Y}$ this pairing induces, as in (9.2), a pairing

$$
\pi_{t}^{\prime} \mathrm{X}^{s} \wedge \pi_{t^{\prime}}^{\prime} \mathrm{Y}^{s^{\prime}} \stackrel{\wedge}{\longrightarrow} \pi_{t+t^{\prime}}^{\prime}(\mathrm{X} \wedge \mathrm{Y})^{s+s^{\prime}}
$$

which, as in 9.4 , can be factored through a pairing

$$
\pi_{t}^{\prime} \mathbf{X}^{s} \wedge \pi_{t}^{\prime}, \mathbf{Y}^{s^{\prime}} \stackrel{\wedge^{\prime}}{\rightarrow} \pi_{t+t}^{\prime},\left(X \wedge^{\prime} \mathrm{Y}\right)^{s+s^{\prime}}
$$

where $\mathbf{X} \wedge^{\prime} \mathbf{Y} \subset \mathbf{X} \wedge \mathbf{Y}$ has the obvious meaning, and one has, as in 9.3 and 9.5,

10.4. Theorem. The pairings (10.2) and (10.3) induce pairings of spectral sequences

$$
\begin{array}{ll}
E_{r}^{s, t} \mathrm{X} \wedge E_{r}^{s^{\prime}, t^{\prime}} \mathrm{Y} \stackrel{\wedge}{\longrightarrow} E_{r}^{s+s^{\prime}, t+t^{\prime}}(\mathrm{X} \wedge \mathrm{Y}), & 1 \leq r \leq \infty, \\
E_{r}^{s, t} \mathrm{X} \wedge E_{r}^{s^{\prime}, t^{\prime}} \mathbf{Y} \wedge^{\prime} E_{r}^{s+s^{\prime}, t+t^{\prime}}\left(\mathbf{X} \wedge^{\prime} \mathbf{Y}\right), & 1 \leq r \leq \infty,
\end{array}
$$

which are augmented, bilinear and associative. Moreover the first pairing is commutative (with sign $\left.(-1)^{(t-s)\left(t^{\prime}-s^{\prime}\right)}\right)$.

Proof. The commutativity is easy. The existence and other properties of the first pairing follow immediately from the existence and properties of the second pairing. And the latter, in turn, is a ready consequence of Theorem 9.5 and the observations:

(i) it suffices (8.4) to consider only the cases $\mathbf{X}=\mathbf{D}_{(r, s, t)}, \mathbf{Y}=\mathbf{D}_{\left(r, s^{\prime}, t^{\prime}\right)}$ and $\mathbf{X}=\mathbf{E}_{(q, t)}, \mathbf{Y}=\mathbf{E}_{\left(q^{\prime}, t^{\prime}\right)}$, 
(ii) in the first case the obvious map $\left(\mathbf{X} \wedge^{\prime} \mathbf{Y}\right)^{k} \rightarrow Z\left(\mathbf{X} \wedge^{\prime} \mathbf{Y}\right)^{k}=$ $\left(Z \mathbf{X} \otimes^{\prime} Z \mathbf{Y}\right)^{k}$ induces an isomorpbism of $\pi_{t+t}^{\prime}$, when $k=s+s^{\prime}$ and of $\pi_{t+t}^{\prime}{ }^{\prime}+r-1$ when $k=s+s^{\prime}+r$,

(iii) in the second case this obvious map induces an isomorphism of $\pi_{t+t}$, when $k=-1$ and of $\pi_{t+q+t^{\prime}+q^{\prime}}^{\prime}$ when $k=q+q^{\prime}$.

We end with

10.5. The group case. For any two simplicial groups $B$ and $C$ we denote by $\pi_{t} B \wedge \pi_{t}, C \stackrel{\wedge}{\rightarrow} \pi_{t+t}, F(B \wedge C)$ the composite pairing

$$
\pi_{t} B \wedge \pi_{t^{\prime}} C \stackrel{\wedge}{\longrightarrow} \pi_{t+t^{\prime}}(B \wedge C) \rightarrow \pi_{t+t^{\prime}} F(B \wedge C)
$$

where $B \wedge C \rightarrow F(B \wedge C)$ is the obvious map. Clearly the pairıng nas properties 10.1 (i), (ii) and (iii).

For two augmented cosimplicial simplicial groups $\mathbf{B}$ and $\mathbf{C}$ this pairing induces, as in (10.2) and (10.3), pairings

$$
\begin{aligned}
& \pi_{t}^{\prime} \mathrm{B}^{s} \wedge \pi_{t}^{\prime}, \mathrm{C}^{s} \stackrel{\wedge}{\longrightarrow} \pi_{t+t}^{\prime} F(\mathrm{~B} \wedge \mathrm{C})^{s+s^{\prime}}, \\
& \pi_{t}^{\prime} \mathrm{B}^{s} \wedge \pi_{t}^{\prime}, \mathrm{C}^{s} \stackrel{\bigwedge^{\prime}}{\longrightarrow} \pi_{t+t^{\prime}}^{\prime} F\left(\mathrm{~B} \wedge^{\prime} \mathrm{C}\right)^{s+s^{\prime}},
\end{aligned}
$$

and one has, as in 10.4,

10.8. Theorem. The pairings (10.6) and (10.7) induce pairings of spectral sequences

$$
\begin{aligned}
& E_{r}^{s, t} \mathbf{B} \wedge E_{r}^{s^{\prime}, t^{\prime}} \mathbf{C} \stackrel{\wedge}{\longrightarrow} E_{r}^{s+s^{\prime}, t+t^{\prime}} F(\mathbf{B} \wedge \mathbf{C}), \quad 1 \leq r \leq \infty, \\
& E_{r}^{s, t} \mathbf{B} \wedge E_{r}^{s^{\prime}, t^{\prime}} \mathbf{C} \wedge^{\prime} E_{r}^{s+s^{\prime}, t+t^{\prime}} F\left(\mathbf{B} \wedge^{\prime} \mathbf{C}\right), \quad 1<r<\infty,
\end{aligned}
$$

which are augmented, bilinear and associative. Moreover, the first pairing is commutative (with sign $\left.(-1)^{(t-s)\left(t^{\prime}-s^{\prime}\right)}\right)$.

The proof is similar to that of Theorem 10.4 .

\section{BIBLIOGRAPHY}

1. A. K. Bousfield and D. M. Kan, The homotopy spectral sequence of a space with coefficients in a ring, Topology 11 (1972), 79-106.

2. - Pairings and products in the homotopy spectral sequence, Trans. Amer. Math. Soc. 177 (1973), 319-343.

3. E. B. Curtis, Simplicial homotopy theory, Advances in Math. 6 (1971), 107-209.

4. A. Dold and D. Puppe, Homologie nicht-additiver Funktoren. Anwendungen, Ann. Inst. Fourier (Grenoble) 11 (1961), 201-312. MR 27 \#186.

5. J. P. May, Simplicial objects in algebraic topology, Van Nostrand Math. Studies, no. 11, Van Nostrand, Princeton, N. J., 1967. MR 36 \#5942.

6. E. H. Spanier, Algebraic topology, McGraw-Hill, New York, 1966. MR. 35 \#1007.

DEPARTMENT OF MATHEMATICS, BRANDEIS UNIVERSITY, WALTHAM, MASSACHUSETTS 02154

DEPARTMENT OF MATHEMATICS, MASSACHUSETTS INSTITUTE OF TECHNOLOGY, CAMBRIDGE, MASSACHUSETTS 02139 (Current address of D. M. Kan)

Current address (A. K. Bousfield): Department of Mathematics, University of Illinois at Chicago Circle, Chicago, Illinois 60680 